\title{
Parameter Estimation Using a Particle Method: Inferring Mixing Coefficients from Sea Level Observations
}

\author{
Femke C. Vossepoel \\ Institute for Marine and Atmospheric Research Utrecht (IMAU), and SRON Netherlands Institute for Space Research, \\ Utrecht, Netherlands \\ Peter Jan Van Leeuwen \\ Institute for Marine and Atmospheric Research Utrecht (IMAU), Utrecht, Netherlands
}

(Manuscript received 10 March 2006, in final form 19 June 2006)

\begin{abstract}
This paper presents a first attempt to estimate mixing parameters from sea level observations using a particle method based on importance sampling. The method is applied to an ensemble of 128 members of model simulations with a global ocean general circulation model of high complexity. Idealized twin experiments demonstrate that the method is able to accurately reconstruct mixing parameters from an observed mean sea level field when mixing is assumed to be spatially homogeneous. An experiment with inhomogeneous eddy coefficients fails because of the limited ensemble size. This is overcome by the introduction of local weighting, which is able to capture spatial variations in mixing qualitatively. As the sensitivity of sea level for variations in mixing is higher for low values of mixing coefficients, the method works relatively well in regions of low eddy activity.
\end{abstract}

\section{Introduction}

State-of-the art ocean models give highly realistic representations of the ocean mean circulation. At the same time, the global coverage of sea level observations provides an increasingly accurate proxy for ocean circulation. Still, differences between observations and models, which are too large to be explained solely by observational error point to model errors. A likely cause for the misfit between model and observations is the parameterization of subgrid-scale mixing processes. The representation of mixing processes in coarseresolution ocean models is a key issue in ocean models as it determines their representation of the transport of heat and salt.

Mixing parameters can be estimated with several data assimilation techniques. Navon (1997) addresses the identifiability and stability of adjoint parameter estimation and gives a comprehensive overview of adjoint techniques for parameter estimation through data as-

Corresponding author address: Dr. F. C. Vossepoel, IMAU/ SRON, Princetonplein 5, NL-3584 CC Utrecht, Netherlands.

E-mail: F.C.Vossepoel@phys.uu.nl similation. Eknes and Evensen (1997) apply the representer method to estimate parameters of a onedimensional Ekman model. More recently, Losa et al. $(2003,2004)$ have illustrated the use of sequential importance resampling (SIR) for parameter estimation in ecosystem models. The ensemble Kalman filter has been applied for parameter estimation in an earth system model by Annan et al. (2005). The advantages of SIR over the ensemble Kalman filter for estimating model parameters have been illustrated by Kivman (2003).

The present paper presents a first investigation to the estimation of mixing parameters using sea level data assimilation. As this is a proof of concept, rather than an attempt to make the best possible estimate, we will focus on synthetic experiments, where the "perfect" outcome is known. We will test an ensemble method that estimates globally homogeneous eddy coefficients, and identify the possibilities to extend this method for spatially dependent parameter estimation.

The data assimilation technique used, a particle method, is an a posteriori analysis of Monte Carlo simulations. The advantage of particle methods is that the solution is not subject to assumptions on linearity. This

DOI: 10.1175/MWR3328.1

(C) 2007 American Meteorological Society 
is important, considering the nonlinearity of many oceanic processes. There is also a clear practical advantage: the method requires only forward model integrations, thereby avoiding the construction of an adjoint model as is the case for four-dimensional variational data assimilation (4DVAR). However, a successful estimate is only possible when the ensemble is sufficiently large to determine the probability density of the model.

Thanks to increasing computational resources, applications of particle filtering and smoothing techniques in meteorology and oceanography have gained interest over the last few years (Anderson and Anderson 1999; Miller et al. 1999; Pham 2001; Kim et al. 2003). Until recently, testing of the method has always been performed on low-dimensional models such as the Lorenz63 model (Lorenz 1963). Van Leeuwen (2003) went one step farther, and tested particle filtering on a five-layer quasigeostrophic model. Here, we will apply a particle method to an ocean general circulation model of high complexity. The degrees of freedom being large, we will need to use an ensemble large enough to deal with this state space.

A brief introduction to the estimation of eddy coefficients is given in section 2. The model and assimilation method is described in section 3 . In section 4 a, we test our methodology with synthetic identical twin experiments. To analyze the performance of the method in a more realistic setting, section $4 \mathrm{~b}$ presents an experiment in which the mixing characteristics of the observed ocean state are structurally different from the ensemble integrations. The adaptation to the method to include local weighting is discussed in section 4c. Limitations of the methodology, as well as consequences for future research is discussed in section 5 .

\section{Estimation of eddy coefficients}

Direct observations of mixing are sparse, and translating these to the parameterization of coarse-gridded ocean models is not straightforward. Although many advanced mixing schemes have been proposed (Cox 1997; Gent and Mc Williams 1990; Large et al. 1994), the choice of mixing parameters is still a very subjective one. For reasons of simplicity, and lack of comprehensive observations, mixing parameters are generally chosen to be spatially homogeneous, although observations show that regional differences in mixing characteristics are large. Attempts to infer eddy transfer coefficients from the distribution of eddy potential energy (e.g., Armi and Haidvogel 1982) and from sea level variability (Holloway 1986; Keffer and Holloway 1988; Stammer 1998) have clarified regional differences in mixing characteristics, but the results of these studies have hardly been incorporated in ocean general circulation models.

There is a clear relation between eddy coefficients in an ocean model and modeled (mean) sea level. For high eddy coefficients, the currents are relatively steady, resulting in time-dependent sea level fields with little temporal and spatial variation. Low eddy coefficients imply more turbulence, and higher sea level variability. As a consequence, the time-mean model state with low eddy coefficients will have sea level gradients that are less sharp than in the case of high eddy coefficients. These different responses to differences in eddy coefficients makes mean sea level a possible proxy for modifications in ocean-mixing coefficients.

Holloway (1986), Keffer and Holloway (1988), and Stammer (1998) based their altimeter-derived eddy diffusivity estimates on sea level variability rather than the mean sea level. They were unable to use mean sea level as a proxy, because at that time, geoid errors were in the order of decimeters, too large to determine the sea level relative to a geopotential surface directly from altimetry. However, with the upcoming Global Ocean Circulation Experiment (GOCE) satellite mission, errors in the geoid on typical oceanographic scales will be reduced to centimeter level, making it possible to accurately observe mean sea level with satellite altimetry. This sea level estimate does not only offer a possibility to derive ocean transport and currents, but it can also be used to improve ocean models. A possible application would be to improve the representation of eddy coefficients in ocean models. The obvious way to do this is to make use of data assimilation.

\section{Methodology}

In the following, we will describe the ocean model used and give an outline of the particle method.

\section{a. Ocean model}

The ocean model applied in this study is the Océan Parallélisé (OPA) 8.1 model as described in the OPA manual (Madec et al. 1998). The model has a global domain. The resolution is $2^{\circ}$ with a meridional refinement in the equatorial band, reaching a latitudinal grid spacing of $0.5^{\circ}$ at the equator. The model has 31 levels in the vertical. A free surface formulation is used for sea level (Roullet and Madec 2000). The time step is 1 $\mathrm{h}$ and $40 \mathrm{~min}$. Vertical eddy diffusivity and viscosity coefficients are computed from a 1.5 turbulent closure scheme (Blanke and Delecluse 1993). Enhanced vertical diffusion is applied to remove statically unstable density profiles (Lazar et al. 1999). 
Lateral diffusion is modeled along isopycnals, using a horizontal filtering of the isopycnals prior to the computation of the isopycnal diffusion operator. The filtering avoids the need for a minimum background horizontal diffusion for numerical stability. In addition to this, a Gent and McWilliams (1990) quasi-adiabatic parameterization is used, in which eddy-induced advection is added to the tracer equation to mimic mixing caused by baroclinic instabilities. With these parameterizations, the equation for the evolution of temperature becomes

$$
\frac{\partial T}{\partial t}=-\nabla \cdot(T \mathbf{U})+D^{T}
$$

where $T$ is temperature, $\mathbf{U}$ is velocity, and

$$
D^{T}=D^{v T}+D^{l T}
$$

are the diffusive terms (the superscripts $v$ and $l$ denoting vertical and lateral, respectively). The lateral diffusive term $D^{l T}$ is then given by

$$
D^{l T}=\boldsymbol{\nabla} \cdot\left(A^{l T} \mathcal{R} \boldsymbol{\nabla} T\right)+\boldsymbol{\nabla} \cdot\left(\mathbf{U}^{*} T\right),
$$

where the first term represents the eddy diffusivity, and the second term the eddy-induced mixing. Here $A^{l T}$ is the eddy diffusivity coefficient for temperature, and

$$
\mathcal{R}=\left(\begin{array}{ccc}
1 & 0 & -r_{1} \\
0 & 1 & -r_{2} \\
-r_{1} & -r_{2} & r_{1}^{2}+r_{2}^{2}
\end{array}\right)
$$

is an operator to relate the geopotential to the isopycnal surfaces, $r_{1}$ and $r_{2}$ being the slopes between these two surfaces. In the eddy-induced diffusivity term $\mathbf{U}^{*}=$ $\left(u^{*}, v^{*}, w^{*}\right)$ represents the nondivergent, eddy-induced transport velocity computed following Gent and McWilliams (1990). The evolution of salinity is described in a similar fashion, defining $A^{l S}$ as the lateral eddy diffusivity coefficient for salinity. In the unperturbed integration, $A^{l T}$ and $A^{l S}$ are chosen to be spatially homogeneous, and have the value $2 . \times 10^{3} \mathrm{~m}^{2} \mathrm{~s}^{-1}$.

Lateral mixing of momentum is Fickian with a lateral eddy viscosity $A^{l m}$ of $4 . \times 10^{4} \mathrm{~m}^{2} \mathrm{~s}^{-1}$, reduced in the Tropics where grid spacing is narrower to reach $2 . \times 10^{3}$ $\mathrm{m}^{2} \mathrm{~s}^{-1}$. The lateral mixing terms in the momentum equation $D_{u}^{l \mathbf{U}}$ and $D_{v}^{l \mathbf{U}}$ are modeled similar to the diffusive terms:

$$
D_{u}^{l \mathbf{U}}=\boldsymbol{\nabla} \cdot\left(A^{l m} \mathcal{R} \nabla u\right)
$$

and

$$
D_{v}^{l \mathbf{U}}=\boldsymbol{\nabla} \cdot\left(A^{l m} \mathcal{R} \boldsymbol{\nabla} v\right)
$$

with $R$ given by (4). Details on the implementation can be found in the OPA manual (Madec et al. 1998).
The model is forced with climatological winds from the National Centers for Environmental PredictionNational Center for Atmospheric Research (NCEPNCAR) reanalysis (Kalnay et al. 1996) as well as heat and freshwater fluxes from this dataset. Sea surface temperature is restored to the Reynolds and Marsico (1993) climatology using a 50-day time scale. A weaker Newtonian damping of temperature and salinity is applied poleward of $60^{\circ}$, using monthly fields of sea surface temperature and salinity from Levitus et al. (1998). All model integrations in this paper start from initial conditions that have been generated with a 5-yr-long spinup, and are integrated for the period of $1 \mathrm{yr}$. It is realized that a 1-yr integration is too short for the estimation of eddy coefficients, as it takes more than 1000 $\mathrm{yr}$ for the ocean to reach an equilibrium, but as we focus on the effects in the upper ocean, this should not be a major concern.

\section{b. Particle method}

In the following section, we will present the formulation of the state estimation in terms of probability functions as given by van Leeuwen and Evensen (1996). The particle method applied in this paper has been denoted the direct estimation method of van Leeuwen and Evensen (1996).

Consider the estimation of a true state $\psi$, given the data. For this estimate, a priori error statistics of both model and data are needed. We can formulate the problem in a Bayesian framework using the probability distribution functions (PDFs) of both model and data. Consider the unknown $\psi$ as the value of a random variable $\bar{\psi}$, and the probability density of the data $d$ a conditional distribution density $p_{d}(d \mid \psi)$ of $\bar{d}$, assuming that $\bar{\psi}=\psi$. Furthermore, the model is assumed to give the a priori information needed to determine the density $p_{m}(\psi)$ of $\bar{\psi}$. Using Bayes's theory, we can write the following:

$$
p_{m}(\psi \mid d)=\frac{p_{d}(d \mid \psi) p_{m}(\psi)}{p_{d}(d)}
$$

where subscript $d$ denotes data and $m$ model. The denominator is rewritten as

$$
p_{d}(d)=\int p_{d}(d \mid \psi) p_{m}(\psi) d \psi,
$$

so we need the prior probability density $p_{m}(\psi)$ as well as the probability density of the data $p_{d}(d \mid \psi)$. The latter is assumed to be given by for instance a Gaussian, and the former will be determined from the model.

To determine $p_{m}(\psi)$ from the model, we assume that the model equations describe a first-order autoregres- 
sive or Markov process. The model can then be represented by

$$
d \psi=g(\psi ; c \mathbf{A}) d t
$$

in which $c$ is a random number, constant in space and time, chosen from a uniform distribution, and $\mathbf{A}$ is a vector of model mixing coefficients. The full evolution of the probability density is not easily determined with an ocean model, but only the first few moments are needed (the best estimate and its error variance). Using Monte Carlo techniques, $p_{m}(\psi)$ is represented by an ensemble of $N$ model integrations:

$$
p_{m}(\psi)=\frac{1}{N} \sum_{i=1}^{N} \delta\left(\psi-\psi_{i}\right),
$$

where $\delta$ is the dirac delta function.

Using this representation in Bayes's Eq. (7), several statistics of the posterior probability density $p_{m}(\psi \mid d)$ can be determined. For instance, the minimum variance estimator, which is the mean of this density, is found as

$$
\begin{aligned}
\hat{\psi}= & \int \psi p_{m}(\psi \mid d) d \psi=\frac{\int \psi p_{d}(d \mid \psi) p_{m}(\psi) d \psi}{\int p_{d}(d \mid \psi) p_{m}(\psi) d \psi} \\
= & \frac{\sum_{i=1}^{N} \psi_{i} p_{d}\left(d \mid \psi_{i}\right)}{\sum_{i=1}^{N} p_{d}\left(d \mid \psi_{i}\right)} .
\end{aligned}
$$

It can be shown that the expected value of a general function $h$ is given by

$$
E[h(\psi)]=\frac{\sum_{i=1}^{N} h\left(\psi_{i}\right) p_{d}\left(d \mid \psi_{i}\right)}{\sum_{i=1}^{N} p_{d}\left(d \mid \psi_{i}\right)},
$$

and assuming that the PDF of the data is known, this expression can be used to evaluate the influence of the data. The probability density of the model is given by the collection of ocean states (particles) as provided by the ensemble of model integrations at each point in time. The expected value is now given by

$$
E[\psi]=\frac{\sum_{i=1}^{N} \psi_{i} p_{d}\left(d \mid \psi_{i}\right)}{\sum_{i=1}^{N} p_{d}\left(d \mid \psi_{i}\right)}
$$

and higher-order moments can be obtained easily.
Since the interest of this paper is in the mean ocean state, the model state $\psi$ is in fact the time average of all model states $\psi(t)$. This implies that the optimal ocean state can only be estimated when all model integrations $\psi_{i}$ with $i=1, \ldots, N$ have been performed. Both (synthetic) observations and ensemble ocean states are obtained by time averaging the model integrations.

The expected value is then obtained by weighting each ensemble member with its "distance" to the data using (13). This implies that each ensemble member has weight $w_{i}$ given by

$$
w_{i}=\frac{p_{d}\left(d \mid \psi_{i}\right)}{\sum_{i=1}^{N} p_{d}\left(d \mid \psi_{i}\right)} .
$$

The probability density of the observations is assumed to be known. Instead of using a Gaussian, we apply a Lorentz density as in van Leeuwen (2003). The Lorentz density is given by

$$
p_{d}(d \mid \psi)=\frac{1}{1+\frac{[d-H(\psi)]^{2}}{\sigma^{2}}},
$$

where $H$ is the measurement operator, and $\sigma$ half the full width at half maximum. The reason for using a Lorentz density is that it has a shape very similar to a Gaussian around its peak, but it is much broader away from the peak, which allows more ensemble members to influence the estimate and is more robust against outliers.

The use of the Lorentz density means that the weight assigned to each ensemble member is given by

$$
w_{i}=\frac{1}{W} \prod_{m=1}^{M} \frac{1}{1+\frac{\left[d_{m}-H_{m}\left(\psi_{i}\right)\right]^{2}}{\sigma^{2}}},
$$

with $W$ given by

$$
W=\sum_{i=1}^{N} \prod_{m=1}^{M} \frac{1}{1+\frac{\left[d_{m}-H_{m}\left(\psi_{i}\right)\right]^{2}}{\sigma^{2}}},
$$

where $m=1, \ldots, M$ represents the observations, $i=1, \ldots, N$ represents the ensemble members, and $H_{m}$ is the projection of the model state to the observations.

The inverse estimate of the particle method is thus a weighted sum of ensemble model solutions. In the present paper, the random perturbations in Eq. (9) are applied to the model parameters for lateral diffusivity and viscosity, $A^{l T, S}$ and $A^{l m}$, which remain constant in 
TABLE 1. Overview of model runs in this paper.

\begin{tabular}{llll}
\hline \multicolumn{1}{c}{ Expt } & Weighting & \multicolumn{1}{c}{ Data generated with } & Noise added to observations \\
\hline PAR0.2 & Global & $A^{l}=0.2 \times A_{0}^{l}$ & $5 \mathrm{~cm}$ \\
PAR0.8 & Global & $A^{l}=0.8 \times A_{0}^{l}$ & $5 \mathrm{~cm}$ \\
VPAR & Global & $A^{l T, S}(x, y)$ based on Holloway $(1986)$ & No \\
VPARN & Global & $A^{l T, S}(x, y)$ based on Holloway $(1986)$ & $1 \mathrm{~cm}$ \\
VPAR-LOC & Local & $A^{l T, S}(x, y)$ based on Holloway $(1986)$ & No \\
VPAR-LOCN & Local & $A^{l T, S}(x, y)$ based on Holloway $(1986)$ & $1 \mathrm{~cm}$ \\
\hline
\end{tabular}

time. Alternatively, one could perturb the ocean state, and the boundary conditions, and/or the initial conditions.

\section{Data assimilation experiments}

A number of synthetic experiments has been performed. To test the methodology, identical twin experiments are carried out and presented in section $4 \mathrm{a}$. Section $4 \mathrm{~b}$ describes the performance of the method in a more realistic setting. In section $4 \mathrm{c}$, local weighting is introduced. An overview of the names and settings of the experiments is given in Table 1.

\section{a. Identical twin experiments}

Identical twin experiments are performed with an ensemble of 128 model integrations. The integrations start from the same initial conditions, and have the same boundary conditions and forcing fields. Only the lateral diffusion coefficient $A^{l T}$ in Eq. (3), its salinity equivalent $A^{l S}$, and lateral viscosity coefficient $A^{l m}$ in Eqs. (5) and (6) are different for each ensemble member. In each integration $i$, the coefficients have been perturbed using the following multiplication:

$$
A_{i}^{l T, S}=c A_{0}^{l T, S}
$$

$$
A_{i}^{l m}=c A_{0}^{l m},
$$

where the subscript 0 refers to the default value, and $c$ is a random number between 0 and 1 , which is constant in time and space (values larger than $2.0 \times 10^{3} \mathrm{~m}^{2} \mathrm{~s}^{-1}$ lead to model instabilities). The random numbers have been chosen from a uniform distribution, and the same factor has been applied for the viscosity as for the diffusivity. The resulting spread in sea level for the ensemble is depicted in Fig. 1.

Largest differences between the ensemble members are found in the western boundary currents (WBCs) and in the Antarctic Circumpolar Current (ACC). These are the regions known to have the largest eddy activity. The noisy character of the spread in these highenergy regions is related to the relatively short $1-\mathrm{yr}$ integration period. Values for the rms sea level deviations reach $10 \mathrm{~cm}$. A comparison of the rms sea level difference for values of $c<0.5$ and for values of $c>0.5$ demonstrates that most of the variability finds its origin in the ensemble members with relatively low $c$ (not shown). In the regions where the spread of the ensemble is largest, the sea level is most sensitive to differences in mixing parameterization.

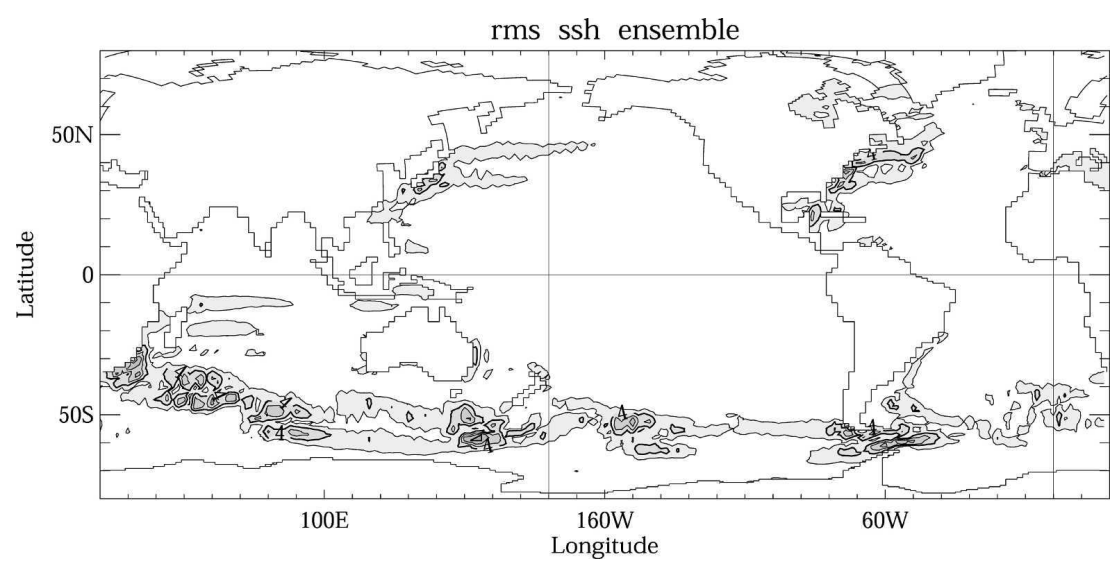

FIG. 1. Rms mean sea level for the 128 -member ensemble. The contour interval is $2 \mathrm{~cm}$. Values greater than $2 \mathrm{~cm}$ are shaded. 
weights PAR0.2

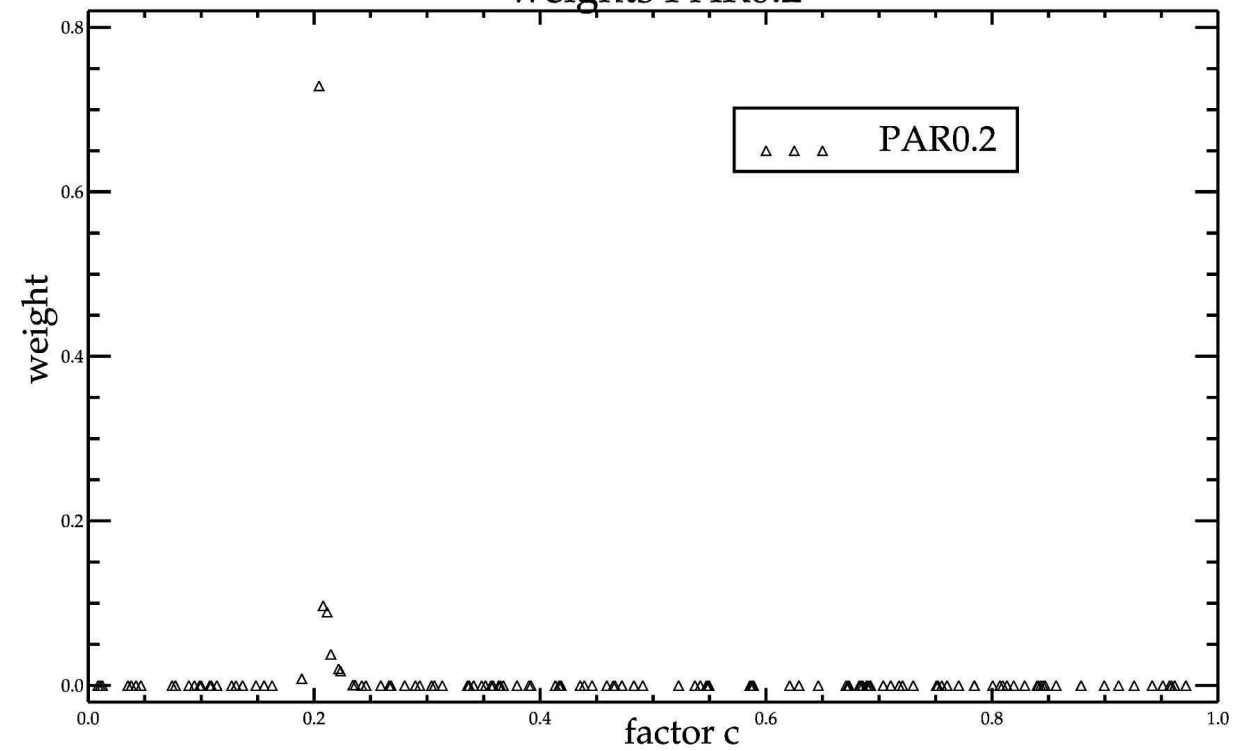

weights PAR0.8

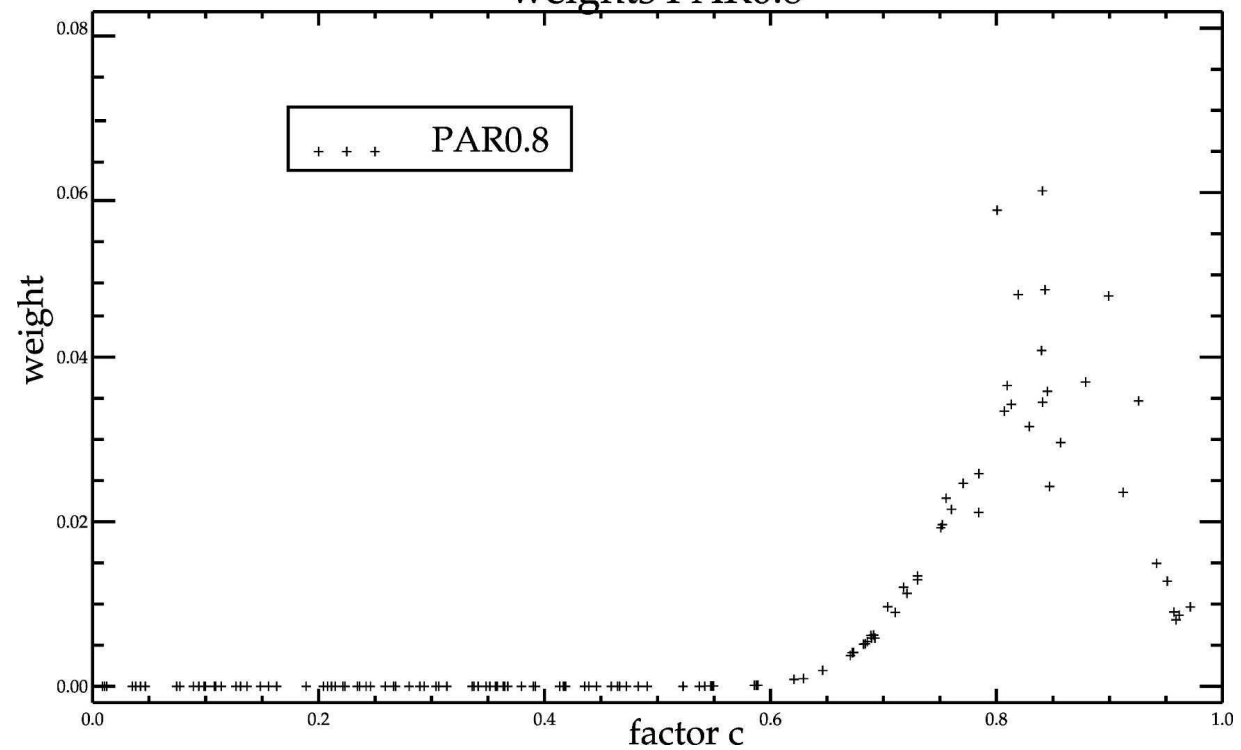

FIG. 2. Values $w_{i}$ for each of the ensemble members as a function of $c$ in PAR0.2 (triangles) and PAR0.8 (plus signs).

Synthetic sea level data are generated with two specific parameter settings. The data for PAR0.2 have been extracted from model output ("truth") with mixing coefficients 0.2 times the default value (so $A^{l T, S}=$ $\left.0.4 \times 10^{3} \mathrm{~m}^{2} \mathrm{~s}^{-1}\right)$, and the data for PAR0.8 with mixing coefficient 0.8 times the default value $\left(A^{l T, S}=1.6 \times 10^{3}\right.$ $\left.\mathrm{m}^{2} \mathrm{~s}^{-1}\right)$. Observations have been obtained for every model grid point, but only one out of four observations has actually been used in the estimation, as spatial correlation is large. Random uncorrelated noise has been added to the observations with an expected value of 5 $\mathrm{cm}$. Accordingly, the $\sigma$ in $(15)$ is chosen to be $5 \mathrm{~cm}$.

Although the setup of the two experiments is the same, the resulting weights $w_{i}$ have a very different distribution. The weights as a function of $c$ are illustrated in Fig. 2. The narrow distribution of weights for PAR0.2 points to a high sensitivity of sea level for mixing when globally the mixing is low. This sensitivity is much less for PAR0.8, as is evidenced by its broad distribution of weights. These results are not surprising 


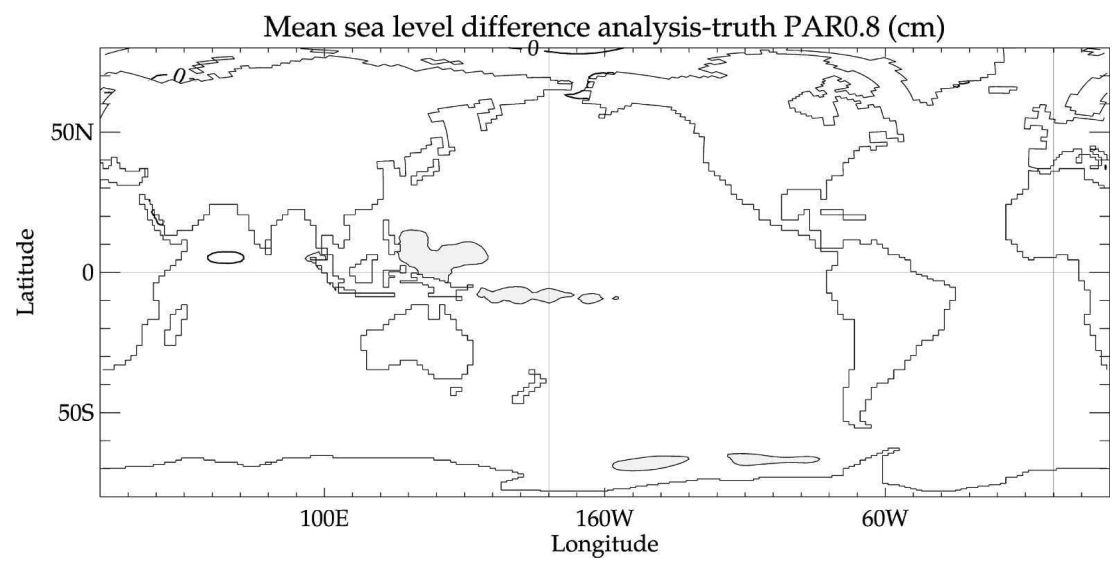

FIG. 3. Difference in mean sea level between the synthetic observations and the inverse estimate of PAR0.8. The contour interval is $1 \mathrm{~cm}$. Differences with an absolute value larger than $1 \mathrm{~cm}$ are shaded, negative differences have dotted contour lines.

considering the fact that most of the spread in the ensemble members originates from the members with low values for $c$.

The PDF of $c$ can be derived from Fig. 2 by counting the number of ensemble members with a certain value for $c$. This procedure is quite sensitive for the binning size that is used in the counting. With the 128-member ensemble, a large binning size is necessary, causing the PDF to be smeared out. Although the PDF is not shown, from the distribution of weights given in this figure it can be concluded that the PDF will be highly nonlinear. The shape of the distribution of weights of PAR0.8 demonstrates the nonlinearity in the relation of sea level and $A^{l}$, even for values of $c$, which are very close to 0.8 . This result indicates that conventional methods such as 4DVAR data assimilation and Kalman filter-like methods would have problems estimating the mixing parameters from sea level observations only. We will discuss this in section 5 .

The resulting sea level in the inverse estimation of PAR0.8 is relatively close to the synthetic truth, as is illustrated in Fig. 3. Difference between estimate and truth remains below the 2 -cm level, which is relatively small, particularly in those regions where the spread in the ensemble is high. In those regions, the difference remains generally below $30 \%$ of the spread (not shown). The reason for this small error, in spite of the 5 -cm noise level, is that the individual ocean states from which the final solution is constructed, are smooth, causing the estimate to be smooth and close to the true field. The difference between the estimate and the noisy observations is around $5 \mathrm{~cm}$, as expected (not shown). The sea level fit for PAR0.2 is similar to the one for PAR0.8, and for that reason not shown.

These results are very encouraging and suggest that indeed eddy coefficients can be reconstructed from sea level observations. The probability density of PAR 0.8 furthermore demonstrate that the sensitivity of sea level for mixing parameters becomes less as mixing parameters are higher in a global sense. In spite of this difference in sensitivity, the reconstructions of sea level in the PAR0.2 and PAR0.8 are equally accurate.

Sea level estimates with an ensemble of 64 members are comparable to those presented here, suggesting that convergence is reached with the 128 -member ensemble.

It should be kept in mind that the synthetic data have been generated with exactly the same model that was used to generate the ensemble. Hence, the truth fields, from which the data are obtained, are fully consistent with the ensemble members. Using real observations, this will not be the case. Moreover, the number of observations will be larger than one per four grid points. Therefore, the following section will discuss a case with observations that are generated with a different model configuration than the ensemble members, using a higher observational density.

\section{b. Experiments with variable mixing coefficients}

In reality, lateral mixing is largely flow dependent. Consequently, a spatially uniform parameterization of mixing, as is commonly applied in ocean models, may not be consistent with the observations. Regional differences in mixing will have an expression in sea level, which will cause local discrepancies between modeled and observed sea level.

To test how the system responds to these discrepancies, we have generated observations from an experiment with a spatially varying mixing parameterization (VPARTRUTH). To obtain this mixing parameterization, we have computed values for the lateral diffusivity 


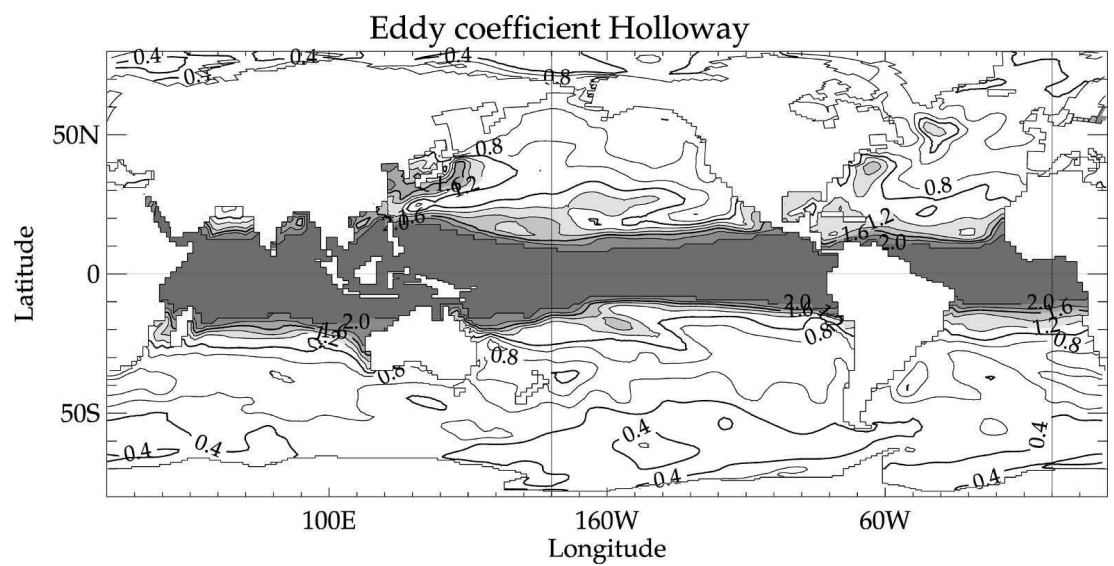

FIG. 4. Lateral mixing coefficient following the approach of Holloway. Contour interval is $0.2 \times 10^{3} \mathrm{~m}^{2} \mathrm{~s}^{-1}$, values greater than $1.2 \times 10^{3} \mathrm{~m}^{2} \mathrm{~s}^{-1}$ are shaded.

coefficient $A^{l T, S}$ following Holloway (1986) and Keffer and Holloway (1988). Their methodology to determine $A^{l T, S}\left(\right.$ or $\left.\kappa_{h}\right)$ is based on the surprisingly simple relation:

$$
A_{\mathrm{VPAR}}^{l T, S}=K \frac{g^{\Xi}}{f},
$$

where $K$ is a constant, $g$ is the gravitational acceleration, $f$ is the Coriolis parameter, and $\Xi$ is the rms sea level variability. The factor $K$, which is largely empirical, is chosen to be 0.12 , in accordance with the results of Stammer (1998). The rms sea level has been determined from monthly sea level fields generated with a 10-yr-long model run for the period 1990-99 of the OPA model (K. Rodgers 2005, personal communication). To prevent singularities at the equator, the maximum value for $A^{l T, S}$ has been set to $2 \cdot 10^{3} \mathrm{~m}^{2} \mathrm{~s}^{-1}$. The resulting values for the lateral diffusivity coefficient are shown in Fig. 4. The general features are similar to the estimates by Stammer (1998): increased diffusivity in the WBCs and near the equator. For consistency, the eddy viscosity $A^{l m}$ in VPARTRUTH has been increased in a similar way as the eddy diffusivity. That is, the default eddy viscosity has been multiplied with a factor of $A_{\mathrm{VPAR}}^{l T, S} / A_{0}^{l T, S}$, where $A_{0}^{l T, S}$ is the default value of the eddy diffusivity coefficient. This way, the gridsize dependency of $A^{l m}$ near the equator is conserved.

Synthetic observations have been sampled from VPARTRUTH at every grid point, and all observations are used in the particle method. The resulting density of the observations (approximately one observation per area of $2^{\circ}$ squared) is more realistic than the density in the PAR0.2 and PAR0.8 twin experiments. The ensemble of model integrations is the same as that which was used in PAR0.2 and PAR0.8, that is, the perturbations are applied on both the $A^{l T, S}$ and the $A^{l m}$ coeffi- cients, and are homogeneous. This time, a $\sigma$ value of 1 $\mathrm{cm}$ is applied in the weighting function in (15). This is the relatively low noise level for sea level that is to be expected when a GOCE-derived geoid becomes available. The resulting analyses are denoted VPAR for the case without noise added to the observations and VPARN for the case where random noise has been added to the observations with a $1 \sigma$ value of $1 \mathrm{~cm}$ (see Table 1).

As there is no spatial variation in the ensemble coefficients, a perfect match with the observations is not possible. Both for VPAR and VPARN, only few members have a nonzero weight, and only one member has a weight that is significantly different from zero. This means that the ensemble fails to represent the model PDF because the ensemble size is too small for this specific problem.

It should be noted that the values for $\Pi_{m=1}^{M} 1 /\{1+$ $\left.\left[d_{m}-H_{m}\left(\psi_{i}\right)\right]^{2} / \sigma^{2}\right\}$ in Eq. (16) become very low because of the large amount of data, the small $\sigma$ value, and the relatively large sea level differences. To deal with this in VPAR, the weights have been normalized. This has been done by multiplying all weights with $1 \times$ $10^{300}$ every time the smallest weight reaches the smallest numerical value possible on the machine. Owing to the small weights in VPAR, this multiplication has been performed 12 times during the course of the weight computation. For VPARN this normalization was performed 19 times. As the normalization is applied to all ensemble members, it has no impact on the final estimate and the corresponding misfit values. The member with a nonzero weight is the same in both estimates, and has a $c$ of 0.29 . This suggests that the weight of each ensemble member is based on its fit in regions of low eddy activity. 


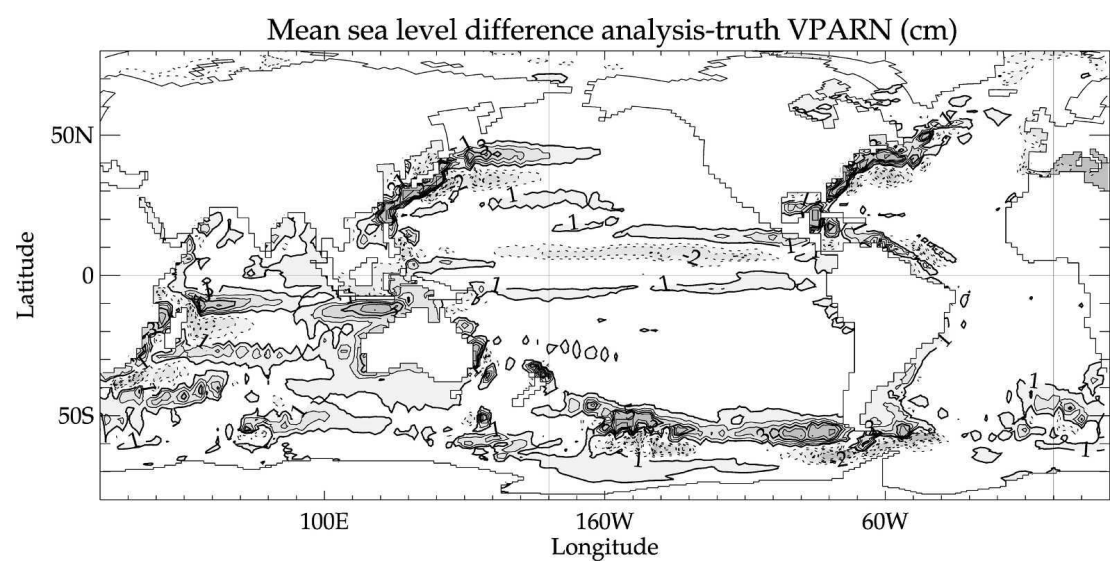

FIG. 5. Difference in mean sea level between the VPARN estimate assimilating VPARTRUTH sea level observations and VPARTRUTH. Noise has been added to the observations with a $1 \sigma$ value of $1 \mathrm{~cm}$. The contour interval is $1 \mathrm{~cm}$.

Figure 5, which depicts the fit of the VPARN mean sea level estimate with VPARTRUTH, illustrates that the method cannot resolve the full complexity of the mean circulation (note that the fit is the same for VPAR and VPARN, as they are both based on the same ensemble member). The differences reach values up to $12 \mathrm{~cm}$ in the WBCs and the ACC. In the open ocean however, the misfit remains below the 2-cm level.

\section{c. Experiments with variable mixing coefficients and local weighting}

As the sea level estimates of VPAR and VPARN have considerable errors in the $\mathrm{WBC}$ regions and the ACC, we wish to take into account spatial variations in the eddy coefficients. The most obvious way to include these spatial variations, is by so-called local weighting. Instead of applying one single weight for each ensemble member, we now determine weights for each grid point. Equation (16) thus becomes

$$
w_{i}(x, y)=\frac{1}{W(x, y)} \prod_{m=1}^{\mathscr{M}} \frac{1}{1+\frac{\left[d_{m}-H_{m}\left(\psi_{i}\right)\right]^{2}}{\sigma^{2}}},
$$

with

$$
W(x, y)=\sum_{i=1}^{N} \prod_{m=1}^{\mathscr{M}} \frac{1}{1+\frac{\left[d_{m}-H_{m}\left(\psi_{i}\right)\right]^{2}}{\sigma^{2}}},
$$

where $\mathcal{M}$ is the number of observations within a $5^{\circ}$ radius of grid point $(x, y)$. The size of this area is chosen such that it is large enough to recover the local gradient from the observations, but the area is small enough to allow for small-scale variations in the eddy coefficients.

The experiments in this section, VPAR-LOC and VPAR-LOCN, are similar to VPAR and VPARN, but have spatially dependent weighting fields instead of global weights. In these experiments, as in VPAR and VPARN, all synthetic observations are used. In this manner, the observations that lie within the area considered for computation of (21) should be sufficient to capture the local structure of sea level.

\section{1) Mean sea level}

The resulting fit of the VPAR-LOC mean sea level estimate to VPARTRUTH is illustrated in Fig. 6. The sea level differences are considerably smaller than those in the VPAR experiment, remaining below the 2-cm level in most of the ocean, including the WBCs. The largest differences occur in the ACC region, but these are still smaller than in the VPAR experiment. The sea level fit for VPAR-LOCN is only slightly affected by the noise in the observations. This is illustrated in Fig. 7.

The differences in performance between the VPAR, VPARN, VPAR-LOC, and VPAR-LOCN experiments are quantified with the rms sea level differences in Table 2. The VPAR-LOC mean sea level fit is more than a factor of 3 times better than the VPAR mean sea level fit.

\section{2) LATERAL DIFFUSIVITY COEFFICIENT}

By construction, VPAR and VPARN estimate one single value for $A^{l T, S}$ and the $A^{l m}$ throughout the basin. For example, the value of $A^{l T, S}$ in the VPAR and 


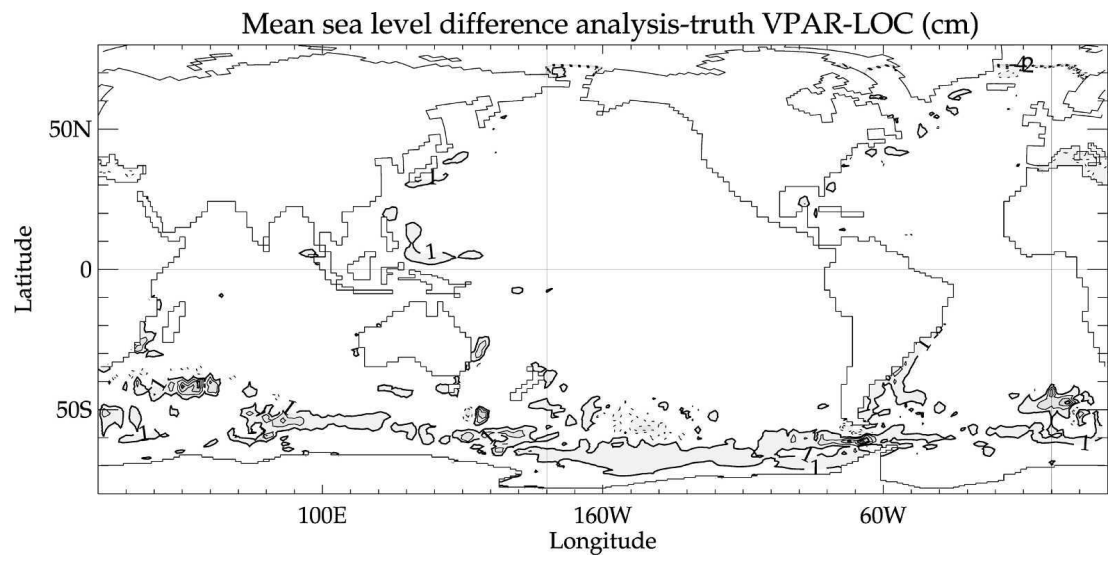

FIG. 6. Difference in mean sea level between the VPAR-LOC estimate and VPARTRUTH. No noise has been added to the observations. The contour interval is $1 \mathrm{~cm}$.

VPARN experiment is equal to that in the ensemble member with nonzero weight: $580 \mathrm{~m}^{2} \mathrm{~s}^{-1}(c=0.29)$. This is lower than the average value of $A^{l T, S}$ in VPARTRUTH, which corresponds to $c=0.45$. The reason for this is that sea level is more sensitive to changes in eddy coefficients in the lower range, than in the higher range, as has been illustrated in section $4 \mathrm{a}$. Thus, a bad fit in regions of low eddy coefficients will be penalized more than a bad fit in regions of high eddy coefficients.

In contrast to VPAR, VPAR-LOC can take into account spatial differences in eddy activity. In the following, we will illustrate this for the eddy diffusivity coefficient $A^{l T, S}$. By construction, the results for the eddy viscosity coefficient are the same.

The discrimination between regions of high and low eddy activity is illustrated for three different points in the estimate in Fig. 8. Two of the three points are chosen in WBC regions: one in the Gulf Stream $\left(38.5^{\circ} \mathrm{N}\right.$, $\left.66^{\circ} \mathrm{W}\right)$, where $A^{l T, S}$ in VPARTRUTH is $1488 \mathrm{~m}^{2} \mathrm{~s}^{-1}$ (corresponding to $c=0.74$ ), one in the Kuroshio $\left(39.8^{\circ} \mathrm{N}, 147.8^{\circ} \mathrm{E}\right)$, where $A^{l T, S}$ in VPARTRUTH is $1729 \mathrm{~m}^{2} \mathrm{~s}^{-1}$ (corresponding to $c=0.86$ ), and one in the open ocean $\left(38.5^{\circ} \mathrm{N}, 34^{\circ} \mathrm{W}\right)$, where $A^{l T, S}$ in VPARTRUTH is $326 \mathrm{~m}^{2} \mathrm{~s}^{-1}$ (corresponding to $c=$ 0.16). Whereas the VPAR experiment only has nonzero weight for $c=0.29$, the VPAR-LOC estimate makes a clear qualitative distinction between regions of very high eddy coefficients, such as the Kuroshio, regions of high eddy coefficients, such as the Gulf Stream, and regions of low eddy coefficients. The exact values of the estimated coefficients, however, are not the same as the true values.

For VPAR-LOCN, the distinction between regions

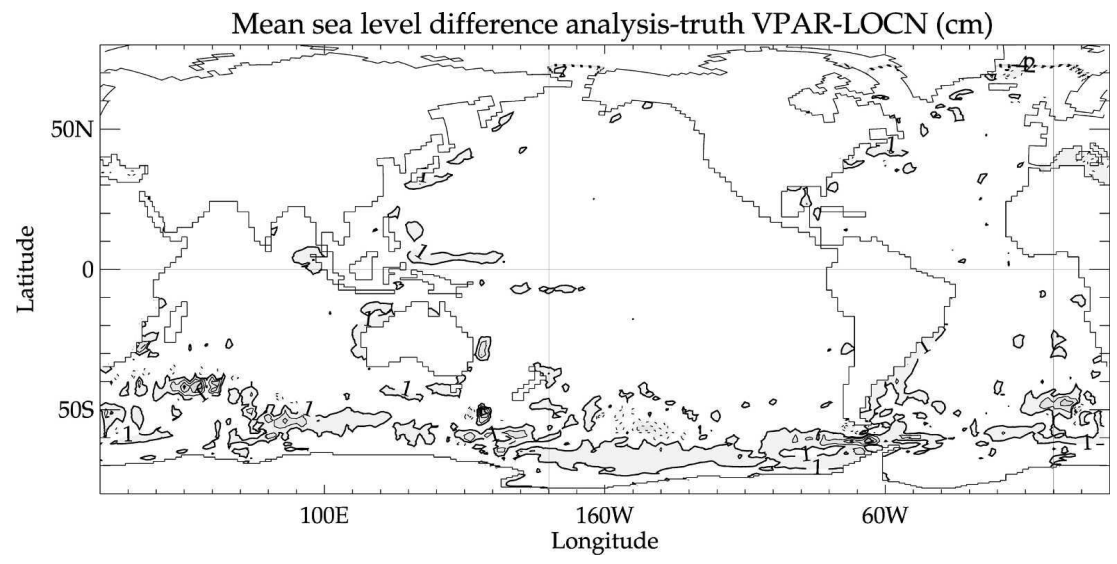

FIG. 7. Difference in mean sea level between the VPAR-LOCN estimate and VPARTRUTH. Noise has been added to the observations with a $1 \sigma$ value of $1 \mathrm{~cm}$. The contour interval is $1 \mathrm{~cm}$. 
TABLE 2. Rms differences of mean sea level for the VPAR, VPARN, VPAR-LOC, and VPAR-LOCN experiments. Values are given in $\mathrm{cm}$.

\begin{tabular}{ll}
\hline \hline Expt & Rms \\
\hline VPAR & 1.46 \\
VPARN & 1.46 \\
VPAR-LOC & 0.41 \\
VPAR-LOCN & 0.45 \\
\hline
\end{tabular}

with different mixing characteristics is very similar to VPAR-LOC, although the distribution of weights is broader. This is shown in Fig. 9. Both in VPAR-LOC and VPAR-LOCN, the coefficients in the Gulf Stream and Kuroshio are underestimated, while the coefficients in the open ocean are overestimated.

Probability density functions in all grid points have been used to determine weighted averages for $A^{l T, S}(x$, $y)$ in VPAR-LOC and VPAR-LOCN. The VPARLOC estimates of $A^{l T, S}$ are depicted in Fig. 10. The estimated $A^{l}$ fields in the VPAR-LOC experiment do not have the same spatial structure as the VPARTRUTH coefficients (cf. Fig. 4). The general tendency of high coefficients near the equator and in WBC regions is well captured, but amplitudes of spatial variations are underestimated (cf. Fig. 4 with Fig. 10). There is a clear increase in $A^{l}$ in the equatorial regions, although there is an equatorial zonal gradient in each ocean basin that is not present in VPARTRUTH. In spite of the sea level misfit in the ACC, the estimate of eddy coefficients in this region is relatively good. The mixing estimate in VPAR-LOCN has a similar spatial structure as in VPAR-LOC, but is considerably affected by noise, as is illustrated in the bottom panel in Fig. 10.

Rms differences of the VPAR-LOC and VPARLOCN estimates of $A^{l T, S}$ with respect to the true $A^{l T, S}$ in VPARTRUTH are lower than in the VPAR(N) experiments, as is presented in Table 3 . The reduction of rms values by a factor of 2 points to a clear improvement in the estimation of eddy coefficients when applying local weighting.

\section{Summary and discussion}

Ocean models are known to have deficiencies in the parameterization of subgrid-scale processes. Data assimilation may help to improve this. Satellite altimetry provides a useful dataset with good global coverage from which parameterizations such as upper-ocean eddy diffusivity and viscosity can in principle be recovered. In the near future, geoid errors will no longer hamper the use of altimetry for mean sea level estimates, as gravity missions such as GOCE will deliver highly accurate data for geoid determination on spatial scales larger than about $100 \mathrm{~km}$. This paper presents the development of a methodology to use these future sea level estimates for the estimation of mixing parameters. The method is first tested in a highly idealized context of homogeneous mixing characteristics. The results of this experiment are promising.

When eddy coefficients are spatially varying, this is not easily captured by the particle method. In the example presented here, the ensemble has failed to represent the required probability density function be-

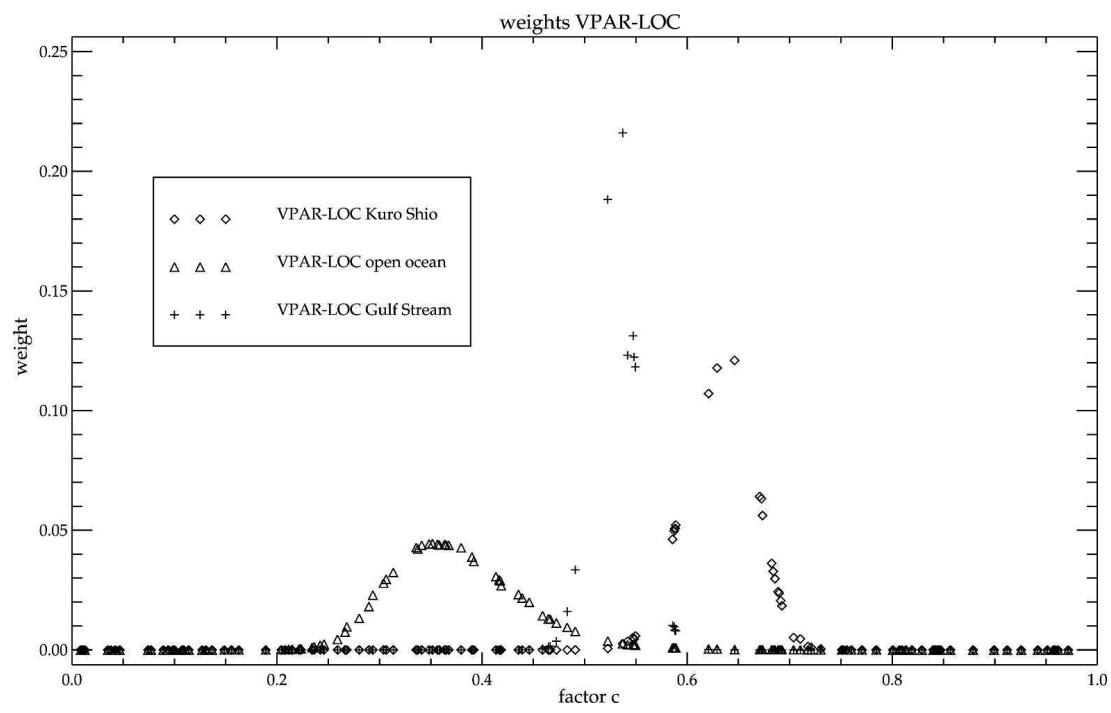

FIG. 8. Weights as a function of $c$ for three different locations in the VPAR-LOC estimate. No noise has been added to the observations. 


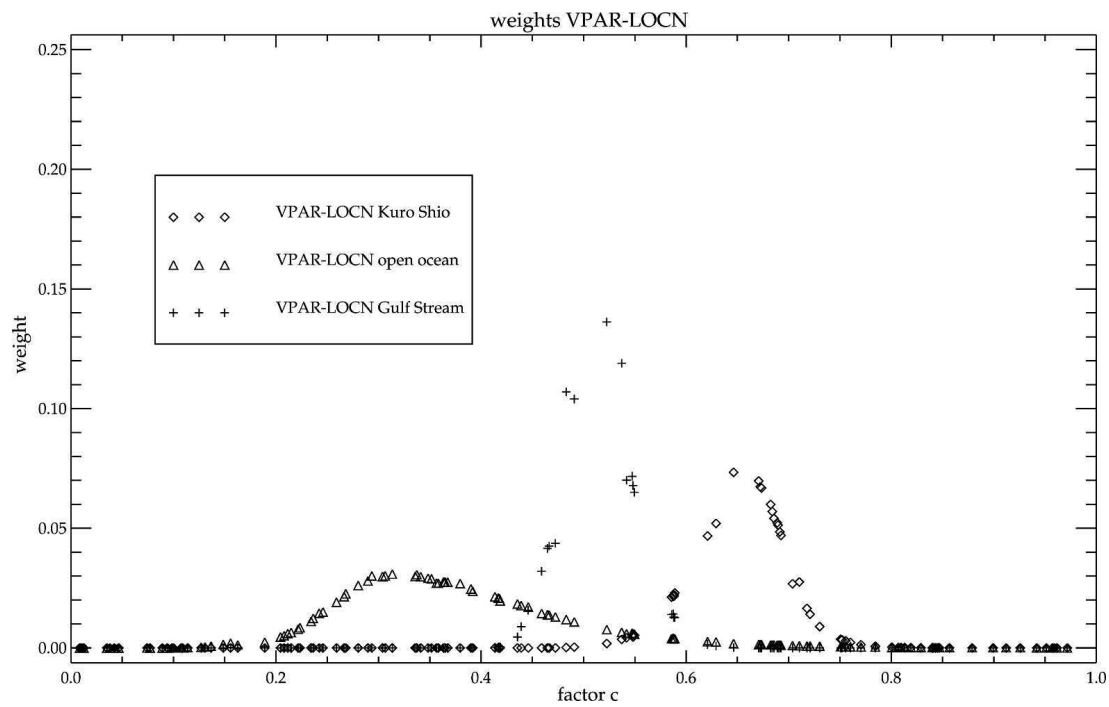

FIG. 9. Weights as a function of $c$ for three different locations in the VPAR-LOCN estimate. No noise has been added to the observations.
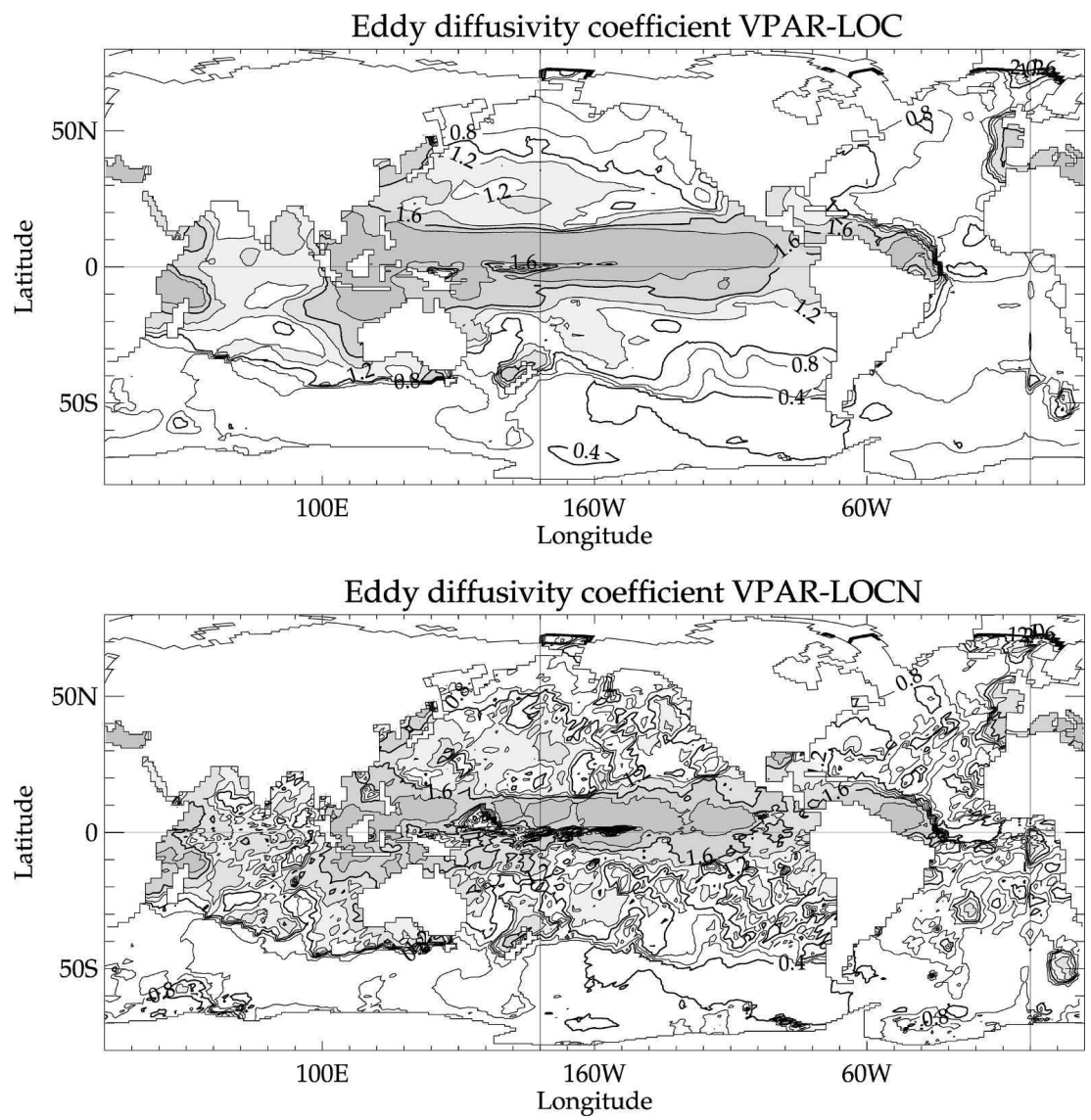

FIG. 10. Estimates for $A^{l}$ based on weighted averages of the $A^{l}$ values in the ensemble members. (top) VPAR-LOC without noise and (bottom) VPAR-LOC with noise added to the observations. The contour interval is $0.2 \times 10^{3} \mathrm{~m}^{2} \mathrm{~s}^{-1}$, values greater than $1.2 \times 10^{3} \mathrm{~m}^{2} \mathrm{~s}^{-1}$ are shaded. 
TABLE 3. Rms differences of $A^{l}$ for the VPAR and VPAR-LOC experiments for both the case of perfect data, and the case of $1-\mathrm{cm}$ random noise added to the synthetic observations. Values are given in $\mathrm{m}^{2} \mathrm{~s}^{-1}$

\begin{tabular}{ll}
\hline \hline Expt & Rms \\
\hline VPAR & 799.6 \\
VPARN & 799.6 \\
VPAR-LOC & 412.8 \\
VPAR-LOCN & 460.4 \\
\hline
\end{tabular}

cause the ensemble size was too small. This has been solved by introducing spatially dependent weighting factors. In this "local weighting" a different combination of ensemble members is made on each grid point of the analysis. By defining a region of influence for each grid point of each ensemble member, we have obtained a spatially coherent solution. Without increasing the ensemble size, this method allows to reconstruct different probability density functions for regions with different mixing characteristics. Comparison of the result to the "ground truth" shows that the particle method with local weighting is able to capture qualitatively spatial variations in mixing coefficients, although further refinement is needed.

It should be noted that the single update approach in the present system is fundamentally different from a sequential approach such as in Kalman filter-like methods or from a variational approach such as in 4DVAR. Moreover, instead of performing a state estimation, the current system attempts to find the parameters that result in a model with the correct climatology.
The present study demonstrates that the probability density function for eddy coefficients given sea level observations is highly nonlinear and particle methods are well suited for that kind of problems. 4DVAR methods are often applied with assumptions on (partial) linearity (e.g., Vossepoel et al. 2004). These assumptions will cause difficulties solving the estimation, because the gradient descent methods used to optimize the solution are unable to deal with multiple minima.

In addition, Kalman filter-like methods will encounter problems when trying to solve for eddy coefficients. The probability density function of sea level can be bimodal as is shown for a point in the Gulf Stream or highly asymmetric as shown for a point in the open ocean in Fig. 11. For PDFs like this, the approximation of the Kalman filter-like methods will result in a broad covariance, from which it can be concluded that the model error is very large, while in fact it is the covariance function that is erroneous. This will result in an estimate that is closer to the observations than it should be (van Leeuwen 2003). Moreover, this may generate a bias in the estimate.

This can be explained when considering Bayes's formalism as given in Eq. (7). In a case where the factor $c$ is varied to find the mixing coefficients that give the best fit in sea level, this equation can be rewritten as

$$
p_{m}(c \mid d)=\frac{p_{d}(d \mid c) p_{m}(c)}{p_{d}(d)}
$$

where $c$ is the random factor used to perturb the mixing coefficients. The likelihood $p_{d}(d \mid c)$ in Eq. (23), which

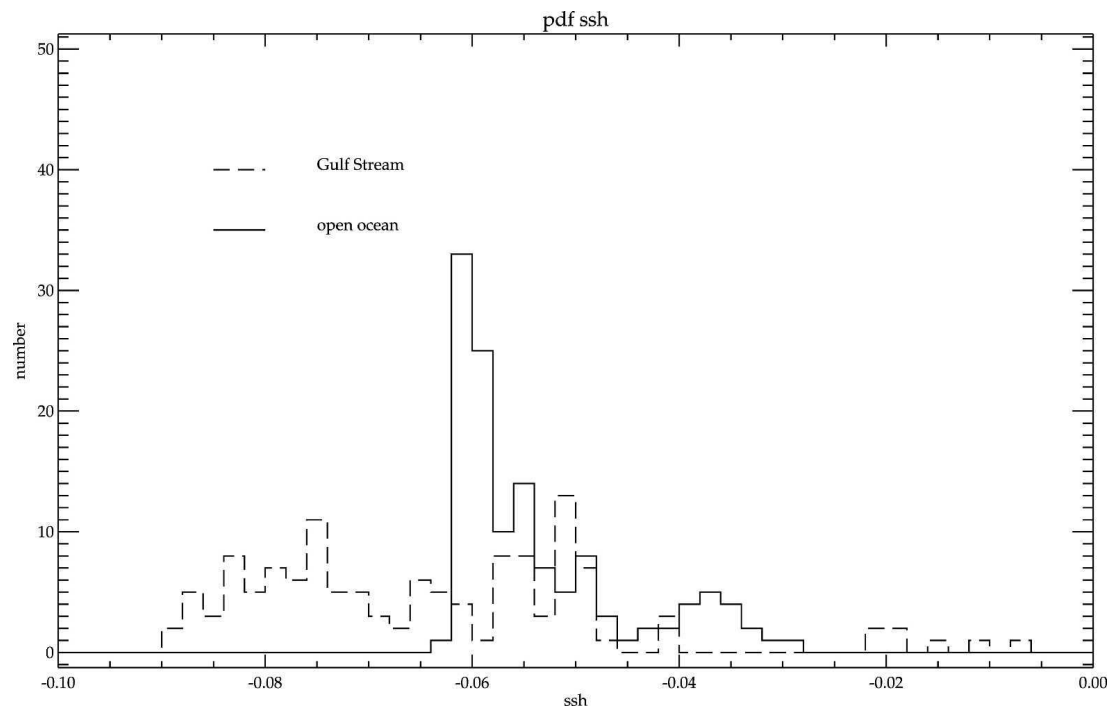

FIG. 11. PDF of sea level for one point in the Gulf Stream (dashed line) and one in the open ocean (solid line). 
can be derived from Fig. 2 as indicated in section 4a, is non-Gaussian. In Kalman filter-like methods, $p_{d}(d \mid c)$ is considered to be

$$
p_{d}(d \mid c)=\exp \left\{-\frac{[d-H(c)]^{2}}{2 \sigma^{2}}\right\} .
$$

The shape of this distribution as a function of $c$ is Gaussian when the measurement operator $H(c)$ is purely linear. In the case of the above experiments, however, the measurement operator is strongly nonlinear, as is demonstrated with the PDF of sea level in Fig. 11. As a result of this, the true shape of the likelihood, that is, $p_{d}(d \mid c)$ as a function of $c$ will be non-Gaussian, and the approximation of Kalman filter-like methods may result in a suboptimal estimate.

Our results suggest that for globally homogeneous mixing parameters, an ensemble size of 128 members is sufficient to determine mixing coefficients from sea level. When mixing is spatially varying, but weighting is global, this ensemble size is too small. Introducing local weighting effectively increases ensemble size, and makes it possible to capture the spatial variations in mixing coefficients. It should be noted that this approximation assumes that information is not propagating in space.

Use of sea level as a proxy for ocean mixing is limited. The spread in our ensemble hardly exceeds the 10-cm level, which implies that we will need highly accurate observations to apply the method presented above. When noise levels are set to $5 \mathrm{~cm}$ instead of 1 $\mathrm{cm}$, the method of local weighting is sensitive to observational noise, resulting in an rms error of $A^{l T, S}$ of $584 \mathrm{~m}^{2} \mathrm{~s}^{-1}$, which is considerably higher than the rms error of VPAR-LOCN, but still smaller than that of VPARN.

Our results demonstrate that the method will not be equally successful for all levels of eddy diffusivity. When globally the eddy diffusivity is small (as is the case in PAR0.2), sea level is very sensitive to changes in mixing. When mixing coefficients are large in a global sense (PAR0.8), this sensitivity becomes less.

The method is also sensitive to regional differences. In regions with large velocities, the spread in the ensemble is relatively large, which points to a high sensitivity of sea level for variations in mixing. In regions of low velocities (and small sea level gradients) the spread is relatively small, making it difficult to discriminate between different solutions, especially when noise is added to the observations. Local weighting successfully reconstructs the general features of the spatial variance in mixing coefficients but fails to capture its full complexity. To properly account for all variations, the method presented above will profit from additional in situ information.

It should be noted that the length of our simulations (1 yr) is in fact too short to obtain a steady ocean circulation. Adaptation of the ocean to modifications in mixing parameters will take several years to decades, especially in the deeper layers. In the upper ocean, however, adjustment time scales are small, and $1 \mathrm{yr}$ will be sufficient to capture the impact of mixing on sea level. For estimations of the full eddy-coefficient field, longer integrations will be needed. It is probable that the success of the method with these longer integrations will be similar to what is shown here.

Of course, lateral mixing parameters are not the only uncertainties in the model. Vertical mixing parameters, but also boundary conditions and surface forcing may introduce considerable errors in the model. To take into account the full range of possible model error, other parameters and model variables will need to be perturbed. As state space will increase, larger ensembles will be needed. The present study is only a stepping stone to build an assimilation system that is able to account for the full range of model errors. The evolution of such a system requires further refinement of the methodology, but this is impossible without accurate and global ocean observations. The future GOCE mission is likely to make an interesting contribution to the observational dataset of the World Ocean, but to make real progress in this field, a continuous supply of highly accurate data will be essential.

Acknowledgments. F. C. Vossepoel is funded by the SRON-UU-DUT Framework Program "Space-based Observation of System Earth." P. J. van Leeuwen is partly supported by the MERSEA project of the European Commission under Contract SIP3-CT-2003502885. This work was sponsored by the Stichting Nationale Computerfaciliteiten [National Computing Facilites Foundation (NCF)] for the use of supercomputer facilities, with financial support from the Nederlandse Organisatie voor Wetenschappelijk Onderzoek [Netherlands Organisation for Scientific Research (NWO)]. We thank K. Rodgers for his model run with the OPA model. We are thankful to G. Evensen and an anonymous reviewer for their valuable comments on our manuscript.

\section{REFERENCES}

Anderson, J. L., and S. L. Anderson, 1999: A Monte Carlo implementation of the nonlinear filtering problem to produce ensemble assimilation and forecasts. Mon. Wea. Rev., 127, 2741-2758.

Annan, J. D., J. C. Hargreaves, N. R. Edwards, and R. Marsh, 
2005: Parameter estimation in an intermediate complexity Earth system model using an Ensemble Kalman filter. Ocean Modell., 8, 135-154.

Armi, L., and D. B. Haidvogel, 1982: Effects of variable and anisotropic diffusivities in a steady-state diffusion model. $J$. Phys. Oceanogr., 12, 785-794.

Blanke, B., and P. Delecluse, 1993: Variability of the tropical Atlantic Ocean simulated by a general circulation model with two different mixed-layer physics. J. Phys. Oceanogr., 23, 1363-1387.

Cox, M., 1997: Isopycnal diffusion in a z-coordinate ocean model. Ocean Modell., 74, 1-9.

Eknes, M., and G. Evensen, 1997: Parameter estimation solving a weak constraint variational formulation of an Ekman model. J. Geophys. Res., 102, 12 479-12 491.

Gent, P. R., and J. C. McWilliams, 1990: Isopycnal mixing in ocean circulation models. J. Phys. Oceanogr., 20, 150-156.

Holloway, G., 1986: Estimation of oceanic eddy transports from satellite altimetry. Nature, 323, 243-244.

Kalnay, E., and Coauthors, 1996: The NCEP/NCAR 40-Year Reanalysis Project. Bull. Amer. Meteor. Soc., 77, 437-471.

Keffer, T., and G. Holloway, 1988: Estimating Southern Ocean eddy flux of heat and salt from satellite altimetry. Nature, 332, 624-626.

Kim, S., G. Eyink, A. Restrepo, and G. Johnson, 2003: Ensemble filtering for nonlinear dynamics. Mon. Wea. Rev., 131, 25862594.

Kivman, G. A., 2003: Sequential parameter estimation for stochastic systems. Nonlinear Proc. Geophys., 10, 253-259.

Large, W. G., J. C. McWilliams, and S. C. Doney, 1994: Oceanic vertical mixing: A review and a model with a nonlocal boundary layer parameterization. Rev. Geophys., 32, 363-403.

Lazar, A., G. Madec, and P. Delecluse, 1999: The deep interior downwelling, the Veronis effect, and mesoscale tracer transport parameterizations in an OGCM. J. Phys. Oceanogr., 29, 2945-2961.

Levitus, S., and Coauthors, 1998: Introduction. Vol. 1, World Ocean Database 1998, NOAA Atlas NESDIS 18, 346 pp.
Lorenz, E. N., 1963: Deterministic nonperiodic flow. J. Atmos. Sci., 20, 130-141.

Losa, S. N., G. A. Kivman, J. Schröter, and M. Wenzel, 2003: Sequential weak constraint parameter estimation in an ecosystem model. J. Mar. Syst., 43, 31-49.

,-- , and V. A. Ryabchenko, 2004: Weak constraint parameter estimation for a simple ocean ecosystem model: What can we learn about the model and data? J. Mar. Syst., 45, $1-20$.

Madec, G., P. Delecluse, M. Imbard, and C. Levy, 1998: OPA 8.1 ocean general circulation model reference manual. Note du Pole de Modelisation 11, Institut Pierre Simon Laplace, 91 pp.

Miller, R. N., E. C. Carter Jr., and S. T. Blue, 1999: Data assimilation into nonlinear stochastic models. Tellus, 51A, 167-194.

Navon, I. M., 1997: Practical and theoretical aspects of adjoint parameter estimation and identifiability in meteorology and oceanography. Dyn. Atmos. Oceans, 27, 55-79.

Pham, D. T., 2001: Stochastic methods for sequential data assimilation in strongly nonlinear systems. Mon. Wea. Rev., 129, 1194-1207.

Reynolds, R. W., and D. C. Marsico, 1993: An improved real-time global sea surface temperature analysis. J. Climate, 6, 114119.

Roullet, G., and G. Madec, 2000: Salt conservation, free surface and varying volume: A new formulation for ocean GCMs. $J$. Geophys. Res., 105, 23 927-23942.

Stammer, D., 1998: On eddy characteristics, eddy transports, and mean flow properties. J. Phys. Oceanogr., 28, 727-739.

van Leeuwen, P. J., 2003: A variance-minimizing filter for largescale applications. Mon. Wea. Rev., 131, 2071-2084.

_, and G. Evensen, 1996: Data assimilation and inverse methods in terms of a probabilistic formulation. Mon. Wea. Rev., 124, 2898-2913.

Vossepoel, F. C., A. T. Weaver, J. Vialard, and P. Delecluse, 2004: Adjustment of near-equatorial wind stress with fourdimensional variational data assimilation in a model of the Pacific Ocean. Mon. Wea. Rev., 132, 2070-2083. 